\title{
A New Spectrum Sensing Scheme Using Overlap FFT Filter-bank for Dynamic Spectrum Access
}

\author{
Takanori SATO $^{\dagger}$ and Masahiro UMEHIRA ${ }^{\ddagger}$ \\ Graduate School of Science and Engineering \\ Ibaraki University \\ 4-12-1, Nakanarusawa-cho, Hitachi-shi, Ibaraki, Japan \\ Email: `10nm674r@hcs.ibaraki.ac.jp, ${ }^{\dagger}$ umehira@mx.ibaraki.ac.jp
}

\begin{abstract}
A periodogram scheme, a multi-taper scheme, and a filter-bank scheme are proposed for spectrum sensing for dynamic spectrum access. The filter-bank scheme is advantageous in hardware implementation since the hardware required for communications can be reused for spectrum sensing. However, it is difficult for the conventional filter-bank scheme to change the detection bandwidth, filter type and frequency channel since the conventional filter-bank is based on time domain signal processing. This paper proposes a new spectrum sensing scheme using overlap FFT filter-bank based on frequency domain signal processing, which can flexibly change the detection bandwidth, filter type and frequency channel. In addition, this paper proposes a windowing method to improve the false-detection performance and confirms its improvement by computer simulation. Furthermore, false-detection and miss-detection performance of the proposed spectrum sensing scheme are evaluated and compared with those of the periodogram scheme according to the roll-off factor of the filter and modulation scheme.
\end{abstract}

Keywords-Dynamic Spectrum Access, Spectrum Sensing, Overlap FFT, Filter-bank, Periodogram

\section{INTRODUCTION}

Towards ubiquitous network society, high capacity and broadband wireless communication systems have been developed, and much more frequency spectrum will be required in near future. In particular, the frequency band less than $6 \mathrm{GHz}$ is suitable for mobile communications because of its relatively small propagation loss in mobile environments. However, most of the frequency bands less than $6 \mathrm{GHz}$ are already occupied by the various existing wireless systems, and it is extremely difficult to secure a newly available frequency band for new wireless systems. On the other hand, it was reported that average spectrum utilization efficiency is not very high because the frequency bands licensed to the existing systems are not well-utilized in terms of space and time [1]. This temporally unused frequency spectrum is called "white space."

Cognitive radio is a technology that observes outside radio environments and adaptively transmits and receives information according to the observed results. Various usage scenarios of cognitive radio have been considered and proposed [2][3]. Dynamic spectrum access is one of the most promising application areas of cognitive radio. This approach aims at the so-called secondary utilization of the abovementioned "white space" in terms of time and geographical location under the condition that the primary systems suffer no harmful interference.

Dynamic spectrum access needs spectrum sensing technologies which can detect correctly whether the frequency band is used by the primary systems. A periodogram scheme, a multi-taper scheme and a filter-bank scheme are proposed for spectrum sensing. The multi-taper scheme can make the side lobe smaller than the periodogram scheme. However, the computational complexity is much larger than the periodogram scheme, thus this scheme is not well-suited to the portable devices. The filter-bank scheme is advantageous in hardware implementation because it can reuse the hardware for communications, and good signal detection performance can be expected since it can maximize SNR (Signal to Noise power Ratio) of the received signals by using the matched-filter if the bandwidth and channel frequency are known [4]. However, it is difficult for the conventional filter-bank using the polyphase filter based on FIR (Finite Impulse Response) filters and FFT (Fast Fourier Transform) to change the detection bandwidth, filter type and frequency channel since the conventional filterbank is based on time domain signal processing.

On one hand, we have proposed the flexible channel access scheme using overlap FFT filter-bank for dynamic spectrum access and also reported the performance analysis of the overlap FFT filter-bank [5][6]. This paper proposes a new spectrum sensing scheme using overlap FFT filter-bank. As the overlap FFT filter-bank is based on frequency domain filtering, it can flexibly change the detection bandwidth, filter type and frequency channel. We assume the basic parameters such as frequency channel and bandwidth of the primary system can be obtained from the data base for dynamic spectrum access. Based on this assumption, this paper evaluates the spectrum detection performance, i.e. false-detection and miss-detection performance, of the proposed scheme under AWGN (Additive White Gaussian Noise) environments by computation simulation. The proposed scheme employs energy detection since it does not need more detailed information of the primary systems such as modulation scheme and signal format and it can be easily implemented.

This paper is organized as follows: Section II describes the conventional spectrum sensing schemes, i.e. the periodogram scheme and the filter-bank scheme. Section III describes the proposed spectrum sensing scheme using the overlap FFT filter-bank. Section IV proposes a windowing method for the proposed scheme to improve the false-detection performance and shows the simulation results. Finally, section V describes the performance evaluation of the proposed spectrum sensing scheme and compares the proposed scheme with the conventional periodogram scheme according to the roll-off factor of the filter and modulation scheme of the received signals of the primary systems.

\section{THE CONVENTIONAL SPECTRUM SENSING SCHEMES}

\section{A. Periodogram scheme}

The periodogram scheme multiplies the time-domain signals by a window function, performs FFT, and detects the power of the concerned frequency band. This scheme detects the energy $S\left(f_{i}\right)$ of the received signals with the center frequency $f_{i}$, where $f_{i}$ is given by 


$$
f_{i}=\frac{1}{T_{s}} \cdot \frac{i}{N}
$$

where $T_{s}$ is the sampling period and $N$ is the number of samples. $S\left(f_{i}\right)$ is given by the following equation:

$$
S\left(f_{i}\right)=\sum_{l=i-\frac{L}{2}}^{i+\frac{L}{2}-1}\left|\sum_{k=0}^{N-1}\left(h(k) x(k) e^{-j 2 \pi \frac{k}{N} l}\right)\right|^{2}
$$

where $L$ is the detection bandwidth, $h(k)$ is a window function, and $x(k)$ is the input signal. In time domain, the input signal $x(k)$ is multiplied with a window function $h(k)$, and converted into the frequency domain signals by FFT. The spectrum sensing output $S\left(f_{i}\right)$ is obtained by summing the power of each frequency component over the concerned frequency bandwidth.

The periodogram scheme can control the side lobe level by using the window function, however the main lobe becomes larger and the frequency resolution becomes lower when the windowing is used. The side lobe level depends on the window function.

\section{B. Filter-bank scheme}

Fig.1 shows the block diagram of the conventional filterbank scheme using the polyphase filter based on FIR filters and FFT, where $E_{0}\left(z^{N}\right) \sim E_{N-1}\left(z^{N}\right)$ are the polyphase FIR filters. Fig.2 shows the frequency response of the filter-bank shown in Fig.1. As shown in Fig.2, the filter-bank is equivalent to $N$ filters which have equally spaced center frequencies. The frequency response of each filter is determined by the impulse response of the polyphase FIR filters, i.e. $E_{0}\left(z^{N}\right) \sim E_{N-1}\left(z^{N}\right)$.

The input signal $x(n)$ passes one of the filters with $i$ th center frequency $f_{i}$ and the output of the filter-bank, $y_{i}(n)$ is obtained in time domain. By summing the power of $y_{i}(n)$, the energy, i.e. the spectrum sensing output $S\left(f_{i}\right)$ is obtained as given by the following equation:

$$
S\left(f_{i}\right)=\sum_{n=0}^{N-1}\left|y_{i}(n)\right|^{2}
$$

In the dynamic spectrum access, a cognitive radio device detects the vacant frequency band and transmits/receives the signals. In order to use multiple channels or multiple bandwidths, multi-carrier transmission/reception capability is required. Therefore, the filter-bank must be flexible enough to change the bandwidth, filter type, frequency channel, and the number of the channels. In the case of the conventional filterbank, it is necessary to re-calculate the impulse response from the required frequency response according to the various bandwidths and filter types. This inflexibility is the disadvantage of the above-mentioned conventional filter-bank.

\section{PROPOSAL OF A NEW SPECTRUM SENSING SCHEME USING OVERLAP FFT FILTER-BANK}

We have proposed the channel access scheme using overlap FFT filter bank which can flexibly change the bandwidth and frequency channel of the transmitting and receiving signals [5]. Taking advantage of the flexibility of the overlap FFT filterbank, this paper proposes a new spectrum sensing scheme using the overlap FFT filter-bank (herein-after, FFT-FB for short). Fig. 3 shows the block diagram of the proposed FFT-FB scheme.

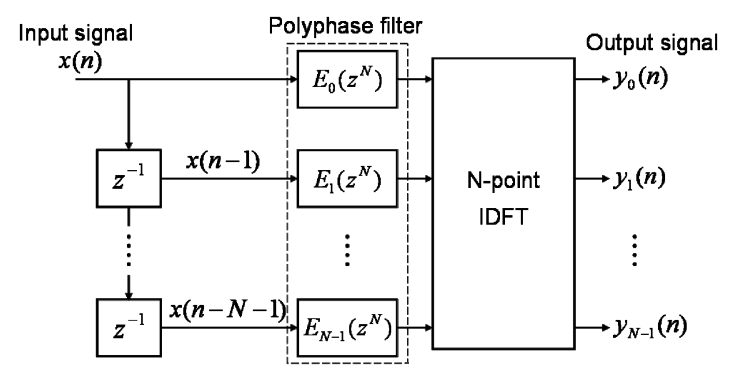

Figure 1. Block diagram of the conventional filter-bank scheme using the polyphase filter and FFT.

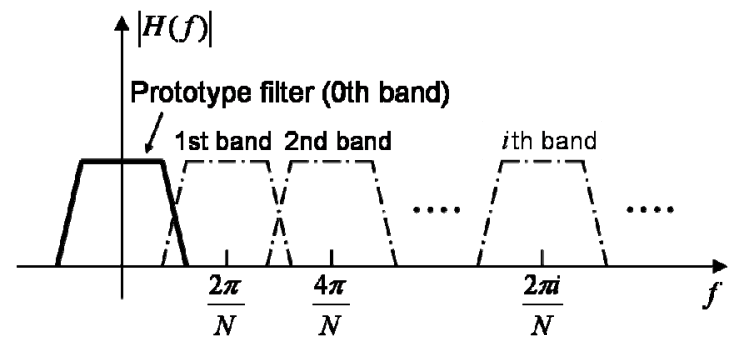

Figure 2. Frequency response of the conventional filter-bank scheme.

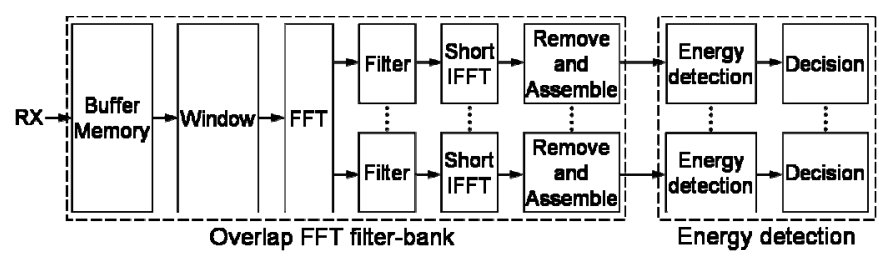

Figure 3. Block diagram of the proposed FFT-FB scheme.

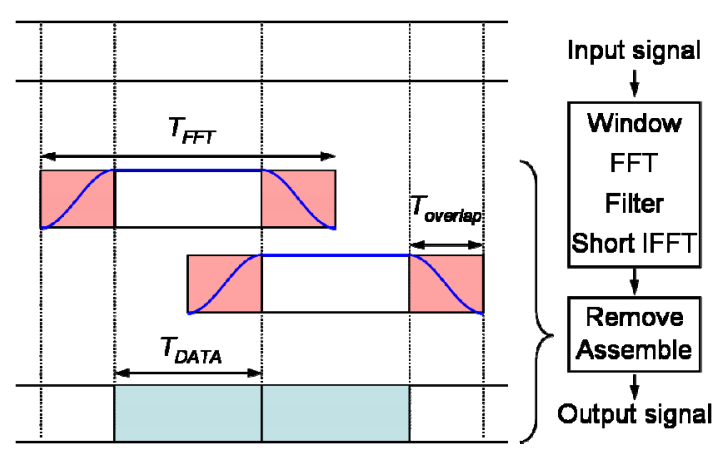

Figure 4. Principle of the overlap FFT processing.

The signal processing in the FFT-FB is as follows: First of all, the block signal including overlap parts is extracted from the received signal stored in the buffer memory. $N$ point FFT is carried out for each divided data to convert the time domain signals to the frequency domain signals. Supposing that the output of the buffer memory is $s(n)(n=0, \ldots, N-1)$, the FFT output, $S_{k}(k=0, \ldots, N-1)$ is given by

$$
S_{k}=\sum_{n=0}^{N-1} S(n) e^{-j 2 \pi \frac{n}{N} k}
$$

After this conversion, frequency domain filtering is performed according to the frequency bandwidth, filter type and frequency channel for spectrum sensing. Then, $M$ point short IFFT is carried out to convert the frequency domain signals to the time domain signals. Let us suppose double samples per 
symbol and the signal bandwidth $B W=M / 2 T_{s}$. The output data from the short IFFT, $g(n)(n=0, \ldots, M-1)$ is given by

$$
g(n)=\frac{1}{M} \sum_{k=i-\frac{M}{2}}^{i+\frac{M}{2}-1} S_{k} R_{k} e^{j 2 \pi \frac{n}{M} k}
$$

Finally, the overlap parts of the time domain signals, i.e. $g(n)$ with $0 \leq n \leq M / 4-1$ and $3 M / 4 \leq n \leq M-1$, are removed and a series of the remained block signals, i.e. $g(n)$ with $M / 4 \leq n<3 M / 4-1$, are assembled as the $M$ sample output signals of the filter. As understood from the above-mentioned signal processing principle, the FFT-FB is able to perform spectrum sensing flexibly since it can directly set the frequency response, bandwidth and frequency channel of the filter for spectrum sensing in frequency domain. For performance evaluation of the proposed scheme, this paper assumes a root raised-cosine roll-off filter. This frequency response, $R_{k}$ of the root raisedcosine roll-off filter is given by

$$
R_{k}=\left\{\begin{array}{cc}
1 & \left(|k|<k_{1}\right) \\
\sqrt{\frac{1}{2}\left(1-\sin \left(\frac{\pi}{2 \alpha} \frac{k-F_{n}}{F_{n}}\right)\right)} & \left(k_{1} \leq|k|<k_{2}\right) \\
0 & \left(k_{2} \leq|k|\right) .
\end{array}\right.
$$

The parameter $k_{1}$ and $k_{2}$ determined the cut-off range are given by $k_{1}=F_{n}(1-\alpha)$ and $k_{2}=F_{n}(1+\alpha)$ where $F_{n}$ denotes the Nyquist frequency and $F_{n}$ is $M / 4(=B W / 2)$.

Fig. 4 shows the principle of the overlap FFT processing in the FFT-FB scheme. When $T_{F F T}$ denotes FFT block length and $T_{\text {overlap }}$ denotes the overlap length, the desired signal length, $T_{D A T A}$ is given by $T_{D A T A}=T_{F F T}-2 T_{\text {overlap }}$. Any roll-off factor can be implemented by the frequency domain filtering. The role of the overlap parts is to reduce the ISI (Inter-Symbol Interference) and ACI (Adjacent Channel Interference) caused by the discontinuity of the FFT block data. Thanks to removing the overlap parts, ISI is removed and the satisfactory filtering performance is achieved. In this paper, we assume $T_{\text {overlap }}=T_{F F T} / 4$. For more details of the overlap FFT filter-bank, please refer [6].

The selectivity performance can be improved by applying a window function to the divided block data of the received signals, as described later in Section IV. We assume the overlap FFT filter-bank is used for wireless communications as well as spectrum sensing. If the windowing is performed for whole of the received signals, it can cause significant ISI, i.e. BER performance degradation. Therefore, the windowing is performed only in the overlap parts which are removed before assembling the signals.

The proposed spectrum sensing scheme finally determines whether the frequency band is occupied, by detecting the energy of the received signals in the sensed channel and comparing it with the pre-determined threshold. As shown in Fig. 3, the energy of each output of the overlap FFT filter-bank is detected and averaged in time and the decision is made, i.e. occupied or vacant.

\section{SELECTIVITY IMPROVEMENT OF THE PROPOSED SCHEME BY WINDOWING METHOD}

\section{A. Selectivity performance}

In order to evaluate the selectivity performance improvement by the proposed windowing method, the selectivity performance of the overlap FFT filter-bank with/without the windowing is evaluated and compared. If the received level of the adjacent channel signals becomes higher, e.g. due to near-far problem, the leakage power from the adjacent channel becomes larger and the false-detection probability becomes higher. Fig.5 shows the detection energy as a function of the normalized guard-band between the sensed channel and the adjacent channel in use. Hanning window is employed for windowing and its windowing function, $h(k)$ is given by the following equation:

$$
h(k)=\left\{\begin{array}{cc}
\frac{1}{2}-\frac{1}{2} \cos \left(\frac{4 \pi k}{N}\right) & \left(0 \leq k<\frac{N}{4}\right) \\
1 & \left(\frac{N}{4} \leq k<\frac{3 N}{4}\right) \\
\frac{1}{2}-\frac{1}{2} \cos \left(\frac{4 \pi(k+1)}{N}\right) & \left(\frac{3 N}{4} \leq k \leq N-1\right) .
\end{array}\right.
$$

Note that the windowing is performed only in the overlap parts. As shown in Fig.5, when the normalized guard-band is larger than 4 , the selectivity performance is improved by using the windowing method as the bandwidth $B W$ becomes narrower. In the case of $B W=16$, the performance is improved more than $15 \mathrm{~dB}$. However, the improvement by the windowing method is not significant if the bandwidth is wide enough. On the other hand, the selectivity performance improvement by the windowing method is less significant if the guard band is less than 4 and the bandwidth, $B W$ is as narrow as 16 . These results show that the proposed windowing method is effective for sensing the narrow bandwidth signals.

\section{B. False-detection and miss-detection performance}

In spectrum sensing, we need to evaluate the probability of false-detection which decides the vacant channel is occupied, and that of miss-detection which decides the occupied channel is vacant. The false-detection and the miss-detection performance of the proposed scheme with/without the windowing method are evaluated and compared. Fig.6 shows the system model for performance evaluation of the proposed scheme where channel 1 is occupied and channel 2 is vacant. We assume the bandwidth of channel 1 is the same as that of channel 2, i.e. $B W(1+\alpha)$. We also assume AWGN environments.

Fig. 7 shows the false-detection probability as a function of the signal level in channel 1 with/without windowing. The threshold was set up so that the false-detection probability is $5 \%$ when only white noise exists. If the signal level in channel 1 becomes larger, the leakage power increases and the falsedetection probability becomes higher. When the signal level in channel 1 is $32 \mathrm{~dB}$, the false-detection probability is improved from 0.15 to 0.05 by using the windowing method. Fig. 8 shows the miss-detection probability as a function of the signal level in channel 1 with the windowing method. Fig. 8 indicates the miss-detection probability is not improved by the windowing method.

The above-mentioned simulation results confirms that the proposed windowing method can improve the false-detection 


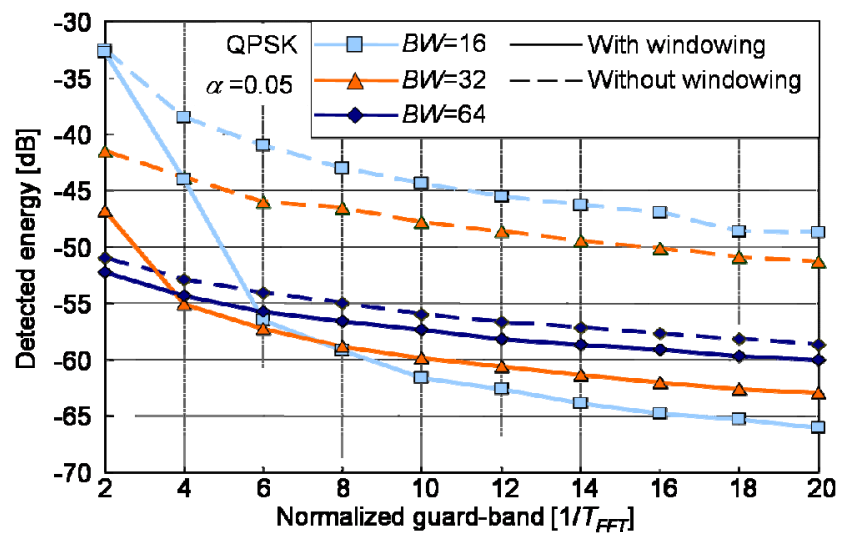

Figure 5. Detected energy as a function of the normalized guard-band.

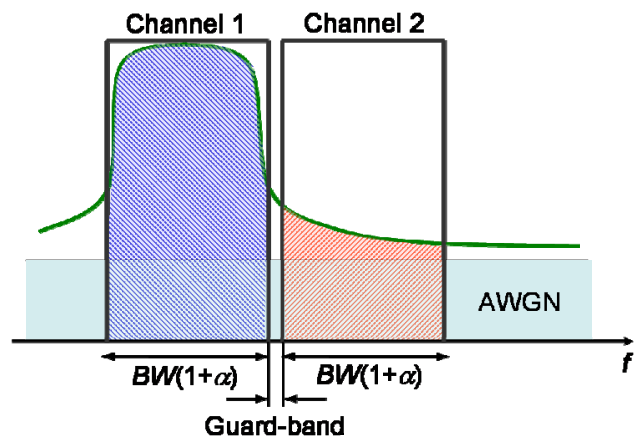

Figure 6. System model for performance evaluation.

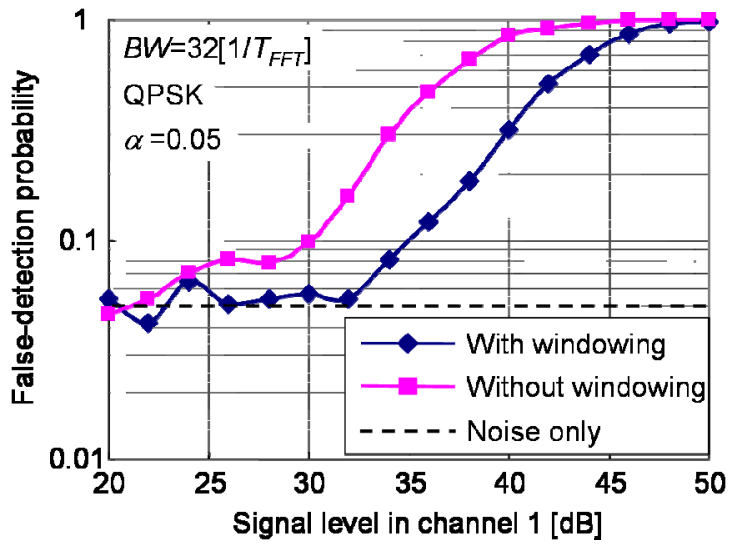

Figure 7. False-detection probability as a function of signal level in channel 1.

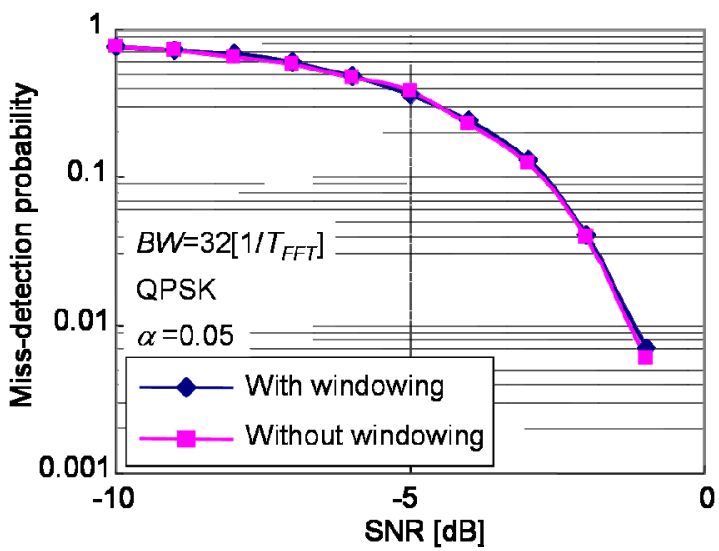

Figure 8. Miss-detection probability as a function of signal level in channel 1 . performance but not the miss-detection performance. Therefore, this paper evaluates the performance of the FFT-FB using the windowing method henceforth.

\section{PERFORMANCE EVALUATION}

\section{A. Simulation conditions}

The simulation parameters for performance evaluation of the proposed FFT-FB scheme are shown in TABLE I. The performance of the proposed scheme is compared with the conventional periodogram scheme. It is assumed that the periodogram scheme uses the same window function as the FFT-FB scheme. After windowing is performed for the received signals, the received signals are converted to the frequency domain signals by FFT, and the received energy is detected by integrating the power of each frequency component over the signal bandwidth in the periodogram scheme.

\section{B. False-detection performance}

\section{1) Effect of the roll-off factor}

Fig.9 compares the false-detection performance of the FFTFB with that of the periodogram scheme as a function of the signal level in channel 1 with a parameter of the roll-off factor. The figure shows both schemes achieves better performances in the case of the roll-off factor, $\alpha=0.5$ than $\alpha=0.05$. The falsedetection performance of the FFT-FB is better than that of the periodogram scheme regardless of the roll-off factor. This is because the FFT-FB has larger out-of-band attenuation than the periodogram scheme, and it has further larger out-of-band attenuation when the roll-off factor is larger.

\section{2) Effect of modulation schemes}

Fig. 10 compares the FFT-FB with the periodogram scheme regarding the false-detection performance as a function of the signal level in channel 1 with various modulation schemes. No significant difference is observed according to the modulation schemes.

\section{Miss-detection performance}

\section{1) Effect of the roll-off factor}

Fig. 11 compares the FFT-FB with the periodogram scheme regarding the miss-detection probability as a function of SNR with the roll-off factor, $\alpha=0.05$ and $\alpha=0.5$. It is shown that both schemes achieve better performance with $\alpha=0.05$ than $\alpha=0.5$. In addition, it is confirmed that the FFT-FB achieves better missdetection performance than the periodogram scheme regardless of the roll-off factor. This is because the periodogram scheme seems to lose the signal power by windowing, while the FFTFB not. In addition, the FFT-FB can maximize SNR by using the matched-filter if the filter-type and the roll-off factor are known.

\section{2) Effect of modulation schemes}

Fig. 12 compares the FFT-FB with the periodogram scheme regarding the miss-detection probability as a function of SNR according to various modulation schemes. It is interesting to see that the miss-detection probability of BPSK and QPSK is worse than that of 16QAM and 64QAM in both schemes. In particular, the worst miss-detection probability for BPSK is smaller than that of the other modulation schemes since the amplitude of the received signals becomes almost zero when modulation data changes. 


\section{CONCLUSION}

This paper proposed a new spectrum sensing scheme using overlap FFT filter-bank, denoted as the FFT-FB scheme. Computer simulations have been carried out to evaluate the performance of the FFT-FB scheme and compare with the conventional periodogram scheme. The simulation results show that the false-detection performance of the FFT-FB scheme significantly improved compared with the conventional periodogram scheme. In particular, the improvement is as large as $15 \mathrm{~dB}$ when $\alpha=0.5$. It is shown that the proposed windowing method decreases ACI and improves the falsedetection performance. In addition, this paper evaluates the false-detection and miss-detection performance of the FFT-FB and compares with those of the conventional periodogram scheme according to the roll-off factor of the filter and the modulation schemes. Future works include performance evaluation of the proposed scheme in fading environments.

\section{REFERENCES}

[1] US Federal Communications Commission Spectrum Policy Task Force, Report of the Spectrum Efficiency Working Group, Nov. 2002, www.fcc.gov/sptf/files/SEWGFinalReport 1.pdf.

[2] J.Mitora III and Gerald Q. Maguire, JR "Cognitive Radio: Making Software Radios More Personal," IEEE Personal Communications, August 1999, pp13-18.

[3] S.Haykin, "Cognitive radio: brain-empowered wireless communications," IEEE JSAC, vol.23, pp.201-220, Feb.2005.

[4] Behrouz Farhang-Boroujeny, "Filter Bank Spectrum Sensing for Cognitive Radios," IEEE TRANSACTIONS ON SIGNAL PROCESSING, VOL. 56, NO. 5, pp.1801-1811, MAY, 2008.

[5] M.Tanabe, M.Umehira, K.Ishihara and Y.Takatori, "A Novel Dynamic Channel Access Scheme Using Overlap FFT Filter-Bank for Cognitive Radio," IEICE TRANS. COMMUN., VOL.E92-B, NO.12, pp.35893596, DECEMBER 2009.

[6] M.Umehira and M.Tanabe, "Performance Analysis of Overlap FFT Filter-bank for Dynamic Spectrum Access Applications," D3S2T3.2 pp.475-479, Proceedings of APCC2010, Auckland, Oct. 2010.

TABLE I. SIMULATION PARAMETERS

\begin{tabular}{|l||l|}
\hline Trial & 10000 \\
\hline Modulation scheme & BPSK, QPSK, 16QAM, 64QAM \\
\hline Roll-off factor $a$ & $0.05,0.5$ \\
\hline Window function & Hanning \\
\hline FFT size $T_{F F T}$ & $2048[$ sample] \\
\hline Bandwidth $B W$ & $64\left[1 / T_{F F T}\right]$ \\
\hline Guard-band & $2\left[1 / T_{F F T}\right]$ \\
\hline Threshold & Set to achieve false-detection probability of 0.05. \\
\hline
\end{tabular}

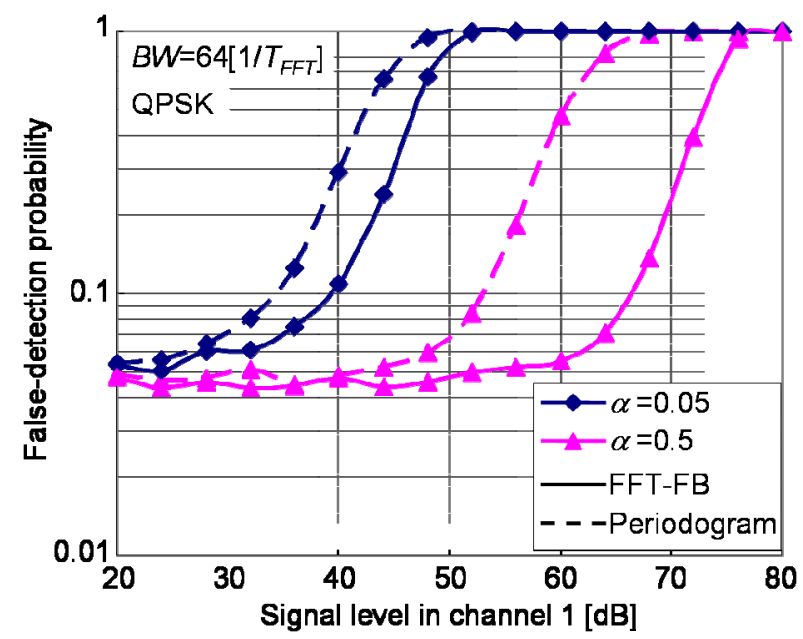

Figure 9. False-detection probability comparison between the FFT-FB and the periodogram scheme as a function of signal level in channel 1 when the roll-off factor, $\alpha=0.05$ and 0.5 .

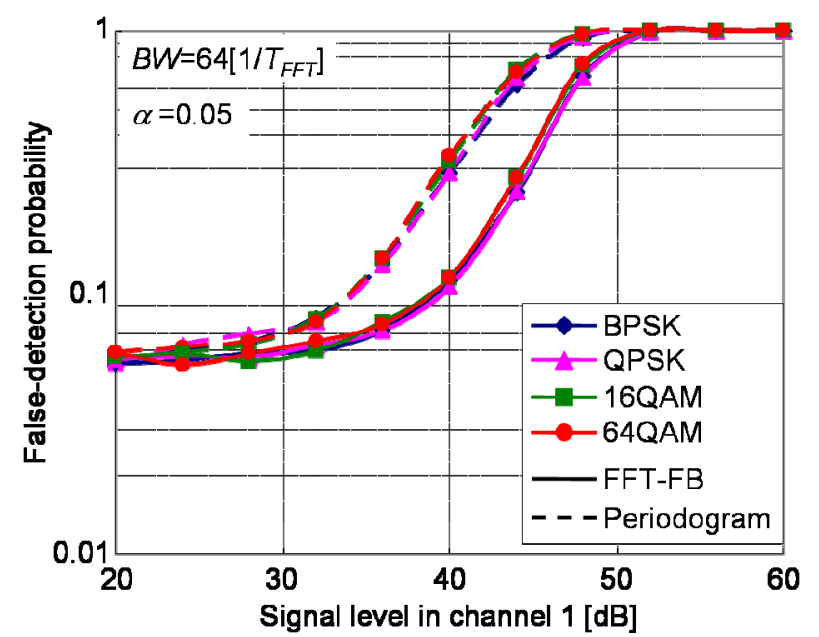

Figure 10. False-detection probability comparison between the FFT-FB and the periodogram scheme as a function of signal level in channel 1 according to modulation schemes.

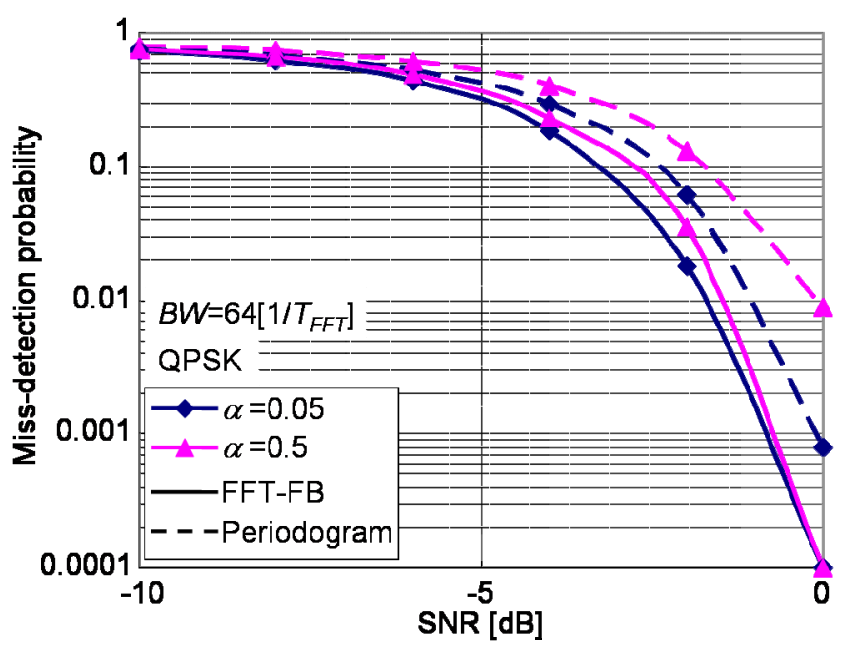

Figure 11. Miss-detection probability comparison between the FFT-FB and the periodogram scheme as a function of SNR when the roll-off factor, $\alpha=0.05$ and 0.5 .

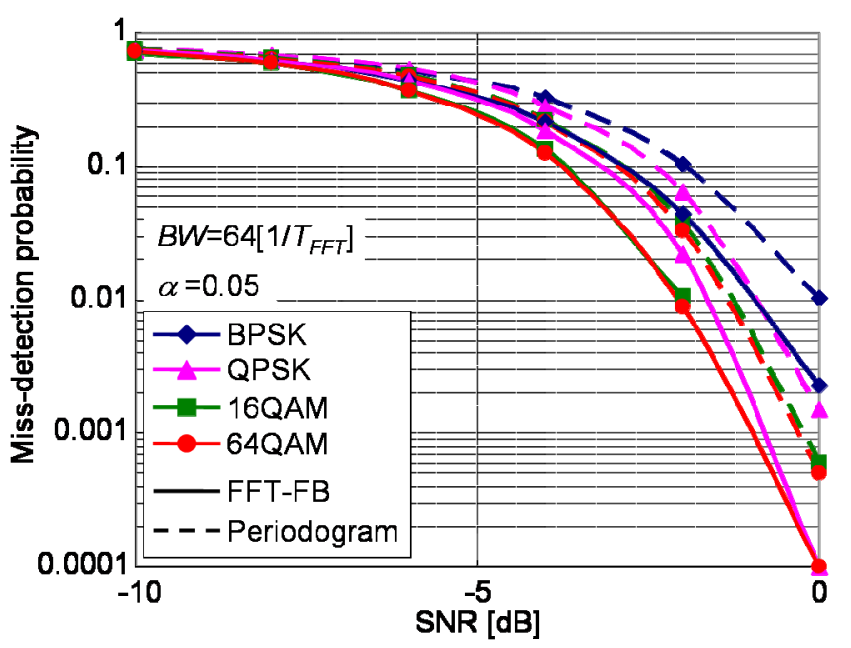

Figure 12. Miss-detection probability comparison between the FFT-FB and the periodogram scheme as a function of SNR according to modulation schemes. 